\title{
20-Year Follow-Up and Reconstruction of a Patient with Craniofacial Microsomia
}

\author{
Rahman $A^{1}$, Kelly $M^{2 *}$, Hegarty $D J^{3}$ and Murray $D J^{4}$ \\ ${ }^{1}$ Medical Intern, National Paediatric Craniofacial Centre, Children's Health Ireland at Temple Street, Dublin 7, Republic of \\ Ireland \\ ${ }^{2}$ Dental Core Trainee, Royal Hallamshire Hospital, Sheffield Teaching Hospitals NHS Trust, Sheffield, United Kingdom \\ ${ }^{3}$ Consultant Orthodontist, St. Finbarr's Hospital, Douglas Road Cork, Republic of Ireland \\ ${ }^{4}$ Consultant Craniofacial, Plastic \& Reconstructive Surgeon, Mater Misericordiae University Hospital, Dublin 7, Republic \\ of Ireland
}

\begin{abstract}
A 20-year-old female patient underwent ligation of her external carotid artery due to life threatening haemorrhagic episodes following a biopsy as a neonate. She was diagnosed with a locally invasive, congenital orbital haemangiopericytoma and subsequently underwent chemotherapy and surgical excision of the tumour. Long term follow-up over 20 years revealed that the patient had developed severe craniofacial microsomia and a Class III asymmetric malocclusion. A two-stage orthognathic surgery was conducted in order to alleviate the functional irregularities and cosmetic appearance. Many patients who present with significant facial difference report aesthetic, psychological and functional benefits following orthognathic surgery.
\end{abstract}

\section{Keywords}

Craniofacial Microsomia, Bimaxillary Osteotomy, External Carotid Artery Ligation, Haemangiopericytoma

\section{Introduction}

Haemangiopericytoma (HPC) is a rare, highly vascular soft tissue sarcoma that was originally described by Stout and Murray in 1942 [1-3]. It is a pervascular tumour that arises in the endothelial lining of capillaries and venules from the surrounding Zimmerman pericytes [1-4]. HPC can be further categorized into infantile and adult forms, due to the more benign character of the infantile form $[5,6]$. Infantile form accounts for all cases that occur before one year of age, most of which are congenital [5]. The adult form refers to all cases that are diagnosed above one year of age [5]. The occurrence of congenital HPC in the Maxilla, orbit, or in the nasal region is rare $[7,8]$. This type of tumour is most commonly located in the lower limbs but can occur anywhere in the body [6]. Although paediatric HPC has been recognized to respond well to chemotherapy treatments, surgical excision has been shown to be the most effective treatment $[5,9]$.

There is a significant post-surgical haemorrhagic risk associated with the excision of HPC in the head and neck region [10-12]. External carotid artery (ECA) ligation is a procedure that is used to prevent or treat post-operative haemorrhage in the areas supplied by this vessel $[13,14]$. By limiting the blood flow to downstream vessels, this procedure helps to significantly reduce and control haemorrhage [15]. The pro- cess of ECA ligation has been thoroughly examined in numerous adult craniofacial procedures and has been shown to be highly effective at preventing post-surgical haemorrhage [1316]. However, long term effects of this procedure on the developing facial structure of infants are unknown.

Craniofacial microsomia (CFM) is a craniofacial developmental disorder which causes facial asymmetry by affecting the growth of head and neck structures derived from the first and second branchial arches $[17,18]$. The disorder causes unilateral under-development of the lower two-thirds of the face, while the other side exhibits normal growth [19]. It is the second most common craniofacial condition after cleft lip and cleft palate $[17,18]$. Although the specific cause for CFM is unknown, a vascular insult to the facial regions has been

*Corresponding author: Dr. Mairéad Kelly, Dental Core Trainee, Royal Hallamshire Hospital, Sheffield Teaching Hospitals NHS Trust, Sheffield, S10 2JF, United Kingdom

Accepted: September 08, 2020

Published online: September 10, 2020

Citation: Rahman A, Kelly M, Hegarty DJ, et al. (2020) 20-Year Follow-Up and Reconstruction of a Patient with Craniofacial Microsomia. J Head Neck Surg 2(1):60-65 
proposed as a possible aetiology for this problem [20].

The primary goal of treatment for CFM is to achieve optimal facial symmetry and facial function [18]. The specific age at which the patient should undergo surgical intervention for this problem is highly debated and varies between institutions $[18,19]$. Optimal facial symmetry and function can be achieved through surgeries such as distraction osteogenesis or orthognathic surgery, to alter the length of the facial bones and ameliorate the facial irregularity [21].

This case report is a follow-up of a patient who was diagnosed with infantile HPC shortly after birth. She was the first reported case as a neonate to have undergone ECA ligation for life-threatening haemorrhagic episodes following a biopsy of the HPC [14]. In this 20 year follow-up, we will examine the long-term sequelae of ECA ligation and HPC resection, including craniofacial microsomia which has become more pronounced with age. This has included combined orthodontic treatment and staged orthognathic surgery.

\section{Case Report}

A 2-week-old baby girl was diagnosed with congenital right orbital and Maxillary HPC. 3D CT revealed that the tumour involved the upper alveolus, the right Maxillary antrum, and the floor of the right orbit (Figure 1 and Figure 2). A biopsy of the buccal mass was obtained on day 20 which confirmed the diagnosis of CHP [14]. Following the biopsy there was persistent and life threatening haemorrhage which required multiple transfusions and surgical interventions including packing and cauterization [14]. Due to the extent and rapid growth of the tumour, and significant concerns of uncontrollable haemorrhage if resected, it was considered that surgi- cal excision was not an option [14]. Vincristine, Adriamycin, and Cyclophosphamide (VAC) chemotherapy was initiated on day 45 of life. Despite treatment with argon therapy, tumour packing and cauterization, the haemorrhage continued and a decision was made for the patient to undergo an emergency ECA ligation [14]. Following a significant reduction in tumour size (as assessed by CT scans) post chemotherapy and ECA ligation, the tumour was surgically excised on day 135 [14]. The patient remained well post-surgery with no signs of recurrence or metastasis and the patient underwent long term prophylactic interferon therapy post-surgery which was completed on July 2000 [14].

CT scan in May 2001 at age 6 showed no changes in a residual soft tissue mass in the right middle cranial fossa with calcification. This mass did not exhibit any increase in size when compared to previous images and no new abnormalities were found. In a follow-up appointment at age 11, there was marked right orbital and maxillary hypoplasia and pronounced craniofacial asymmetry. The patient had abnormalities of the permanent dentition, and a significant malocclusion including a pronounced cant and posterior cross bite. There was also evidence of aplasia in the condylar region of the mandible. The Maxilla showed a significant rotation towards the affected side. Reconstructive surgery was delayed until the patient's facial growth was complete and the patient continued to attend for regular monitoring of her facial growth.

On examination in February 2015, (aged 20 years) the patient was assessed at a craniofacial/orthodontic multidisciplinary clinic and presented with craniofacial microsomia, severe facial asymmetry and poor masticatory function as a

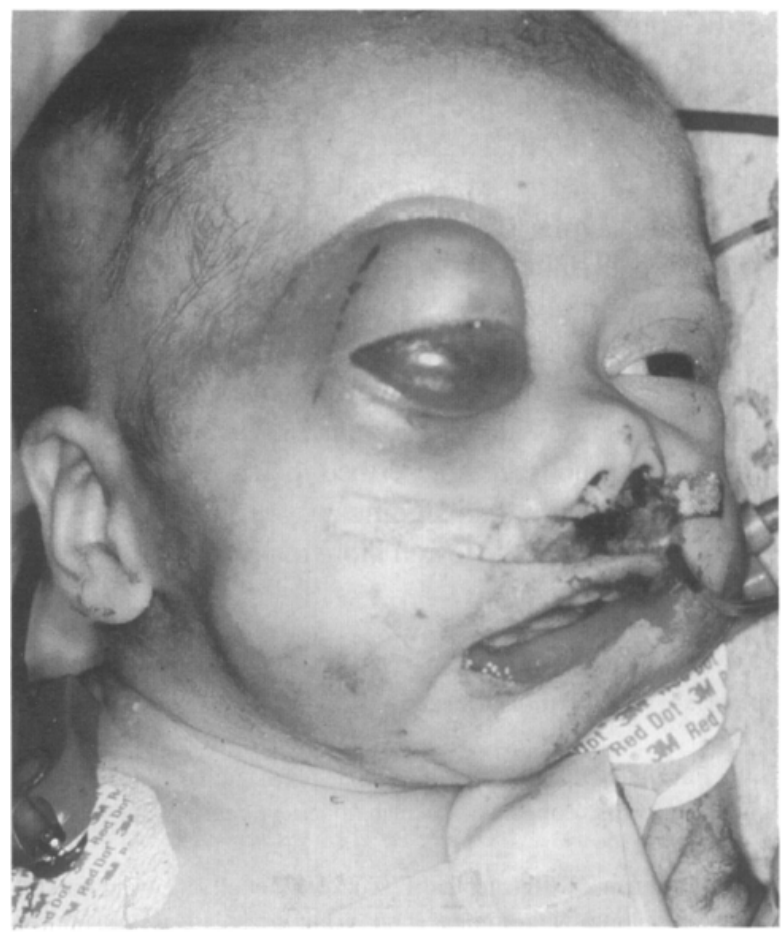

Figure 1

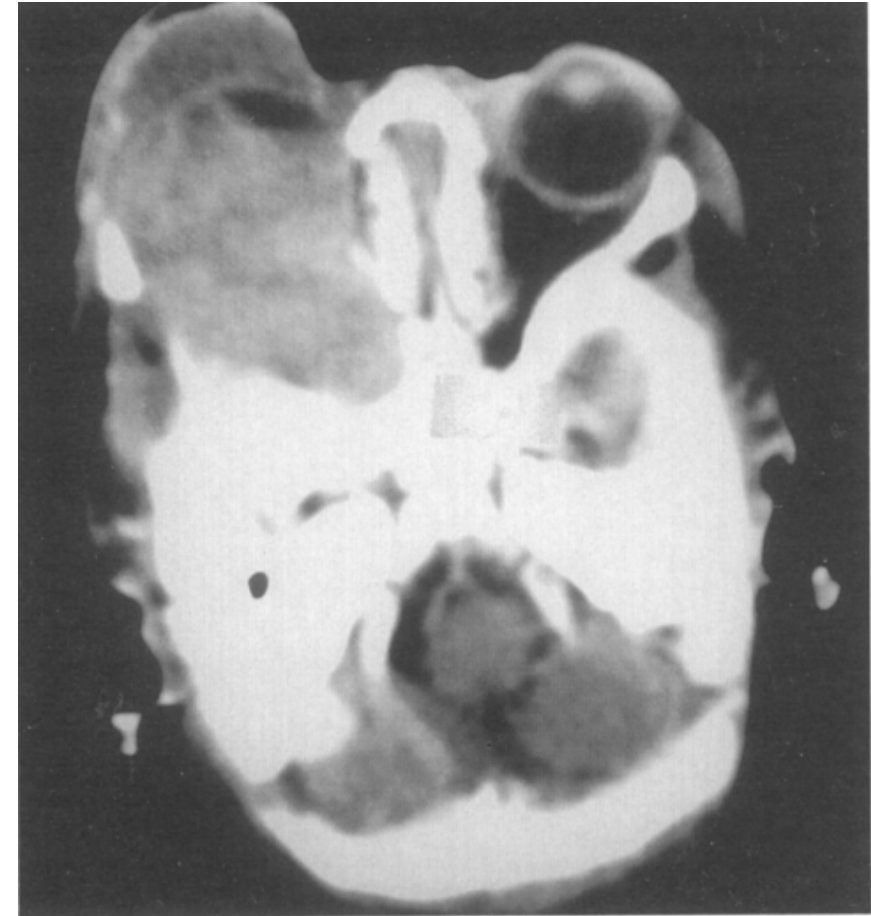

Figure 2

Figure 1 and Figure 2: Preoperative clinical photograph and CT scan, at age 20 days. 
Citation: Rahman A, Kelly M, Hegarty DJ, et al. (2020) 20-Year Follow-Up and Reconstruction of a Patient with Craniofacial Microsomia. J Head Neck Surg 2(1):60-65
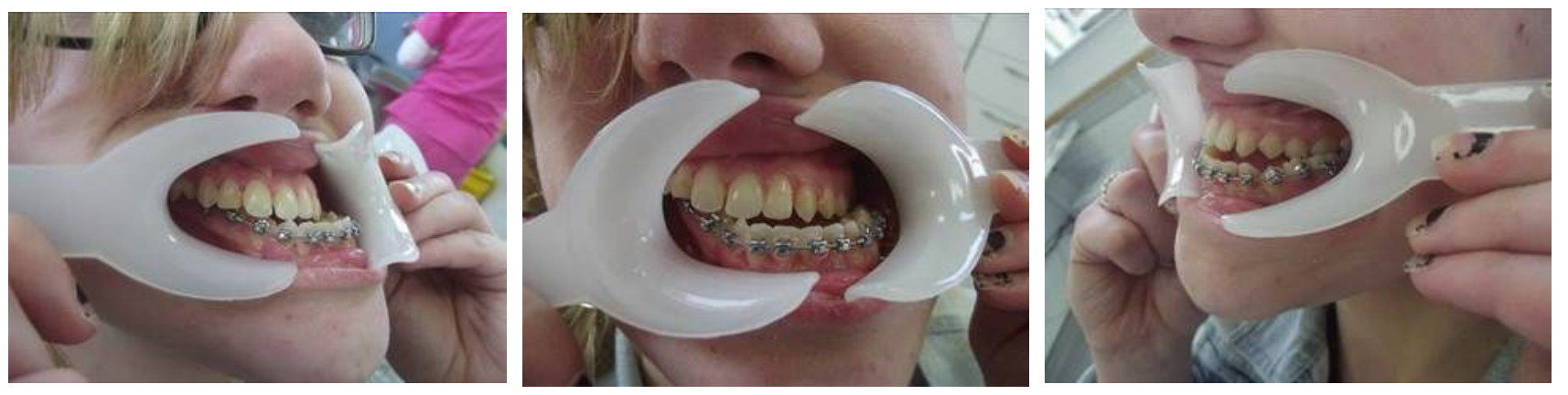

Figure 3: Preoperative dental occlusion, with orthodontic appliances on lower arch.
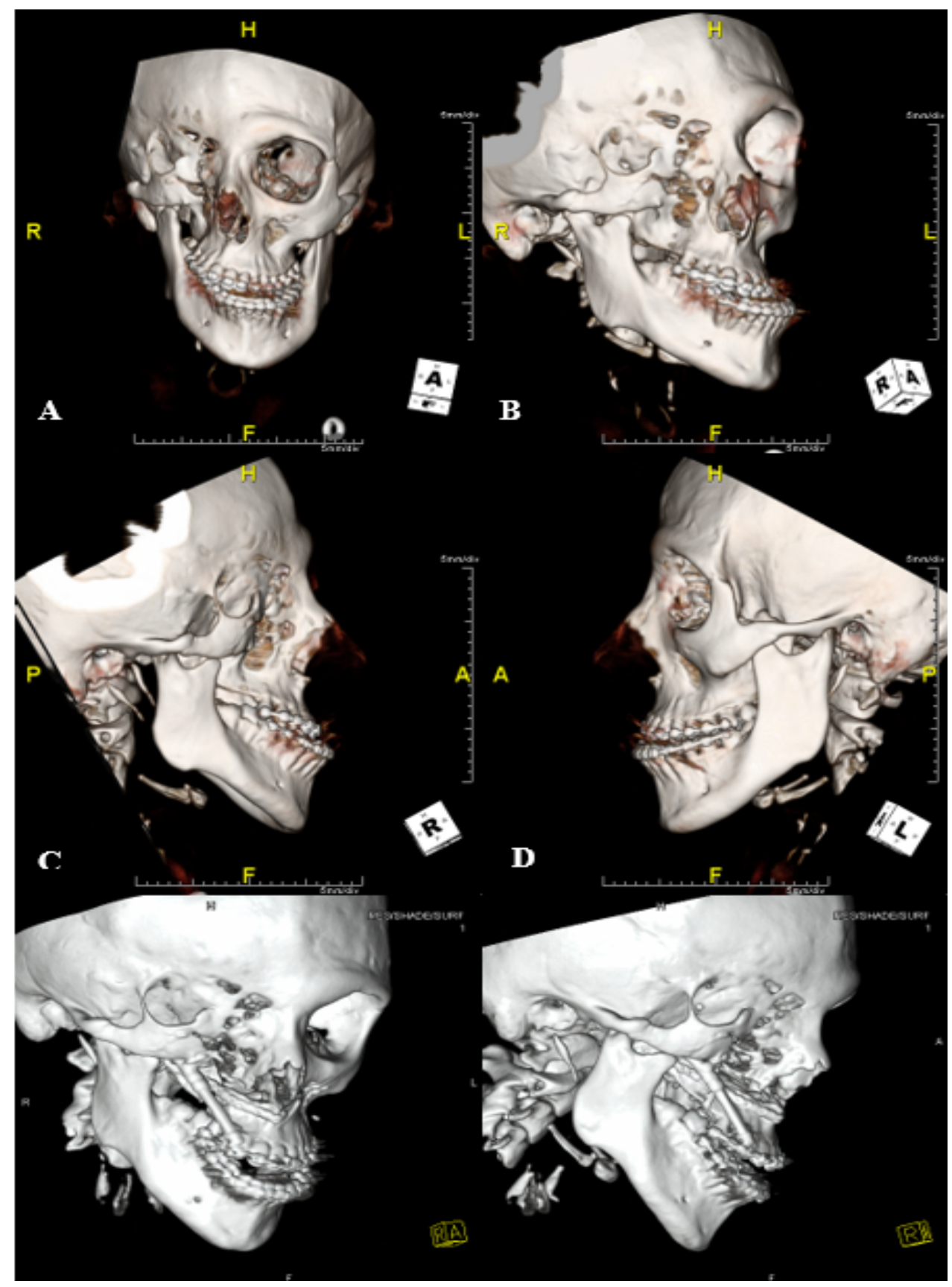

Figure 4: A) AP view of the skull prior to the placement of the Maxillary distractor; B) Oblique view from the right side; C) Side view from the right side; D) Side view from the right side; E) AP view of the skull after the placement of the Maxillary distractor; F) Side view from the right side. 
result of dental malocclusion. Dentally, she had a class III incisal relationship, a class III molar relationship on the hypoplastic right-hand side and a class I molar relationship on the unaffected left-hand side (Figure 3). The patient underwent orthodontic decompensation and arch coordination in preparation for abimaxillary osteotomy.

Orthognathic surgery was planned following facial analysis with photography, 3D CT scan and SAM Articulator Face bow mounted dental models (Figure 4). The bimaxillary osteotomy procedure was initiated 2 years following arch coordination. It was anticipated that it may not be possible to mobilise the Maxilla completely following a Le Fort 1 procedure and it was therefore planned to undertake a distraction osteogenesis of the Maxilla if necessary.

The findings at surgery showed features consistent with hemifacial microsomia; including an asymmetric midface, along with an abnormally dense right Maxilla which was difficult to osteotomise. Subperiosteal dissection was carried out to expose the Maxilla, Piriform Apertures, and the Nasal Cavity. The nasal septum was detached inferiorly. A Maxillary distractor (Zurich Pediatric Maxillary distractor - KLS Martin) was secured to the right hypoplastic Maxillary buttress and the osteotomised Maxilla below the osteotomy line (Figure 5). A flexible titanium plate was placed on the left side connecting the left buttress and the left Maxilla in order to create a 'pivot point' about which the right Maxilla could rotate. 5 $\mathrm{mm}$ of bone was removed for impaction from the left Maxilla to correct the occlusal cant. The distraction of the right Maxilla occurred over a 2 week period where the Maxilla was mobilised $1 \mathrm{~mm}$ per day.

3 weeks later, the patient was readmitted and underwent a 'docking' procedure of the Maxilla into the predicted class 1 incisal and molar occlusions. The Maxilla was also aligned with the midfacial plane and supported with a corticocancellous bone graft from the iliac crest. This was followed by a Bilateral Sagittal Split Osteotomy of the mandible to complete the ideal class I occlusal alignment. The patient was discharged 3 days post-surgery in elastic traction to finalise the occlusion over a period of 6 weeks. The patient made an uneventful recovery and the orthodontic appliance was removed 9 months after surgery (Figure 6a, Figure 6b, Figure 6c and Figure 6d).

\section{Discussion}

Congenital HPC is a rare, soft tissue sarcoma that can be found anywhere in the body [1-4]. Chemotherapy treatments have been shown to be effective in its treatment however, total surgical excision of the tumour remains the definitive treatment for the disease [5,9]. HPC is highly vascularized and both surgical excision and diagnostic biopsies are associated with a high risk of bleeding episodes [10-12]. In paediatric cases, such bleeding can be life threatening and can require extensive procedures such as embolization or ligation of the supplying arteries, cauterizing the smaller blood vessels, or mechanical obstruction of the bleeding by packing the area with gauze [10-12]. In this case report, the patient had multiple haemorrhagic episodes post biopsy which required the ligation of her right ECA.

The use of chemotherapy in conjunction with surgery has been shown to be a more effective method of treatment for HPC than surgery alone [7]. However, exposure to adjuvant therapies to treat head and neck HPC, particularly in newborn patients increases the risk of severe complications over periods of time [7]. These complications include impedance in facial growth, haemorrhagic risk, decreased wound healing, and secondary malignancy [7].

We believe that the ligation procedure at such a young age may have played a significant role in the patient developing craniofacial microsomia later in life. Craniofacial microsomia has been associated with decreased blood flow to the facial regions during embryonic development at approximately one month of gestation [20]. Although, the ECA ligation was carried out after birth, it is postulated that the severe vascular insult could still impact significantly on bone and tissue growth. Although the effects of the surgery and chemotherapeutic agents cannot be discounted, the extensive area involved suggests an extensive vascular insult. The
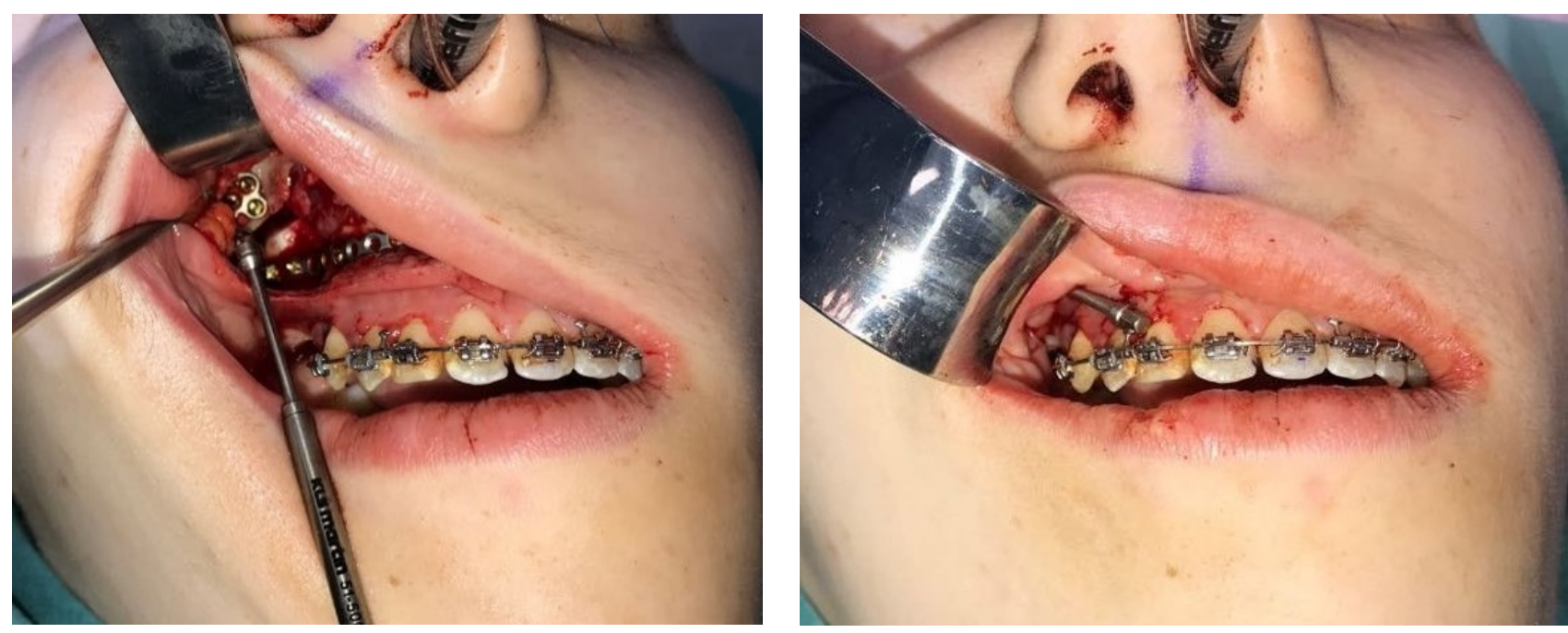

Figure 5: Intraoperative clinical photographs, with distractor in situ. 
Citation: Rahman A, Kelly M, Hegarty DJ, et al. (2020) 20-Year Follow-Up and Reconstruction of a Patient with Craniofacial Microsomia. J Head Neck Surg 2(1):60-65

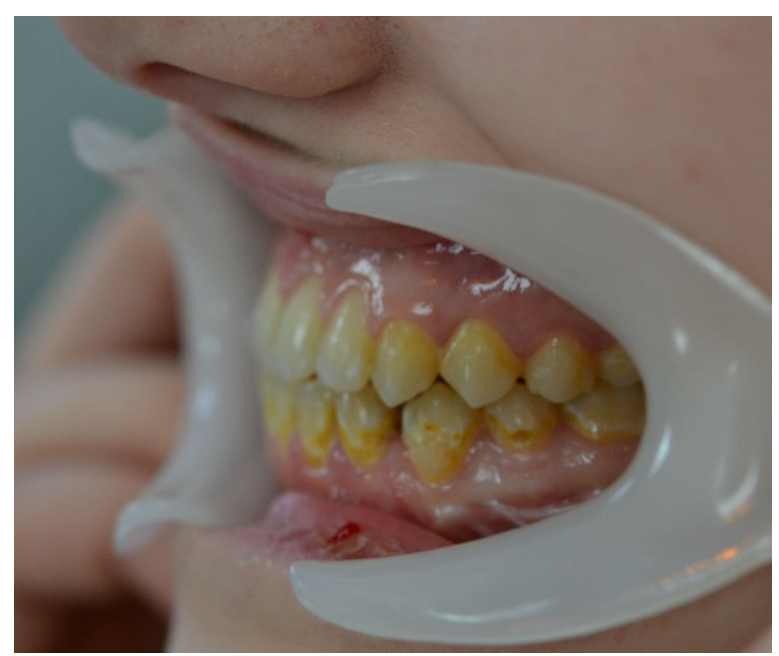

Figure 6a

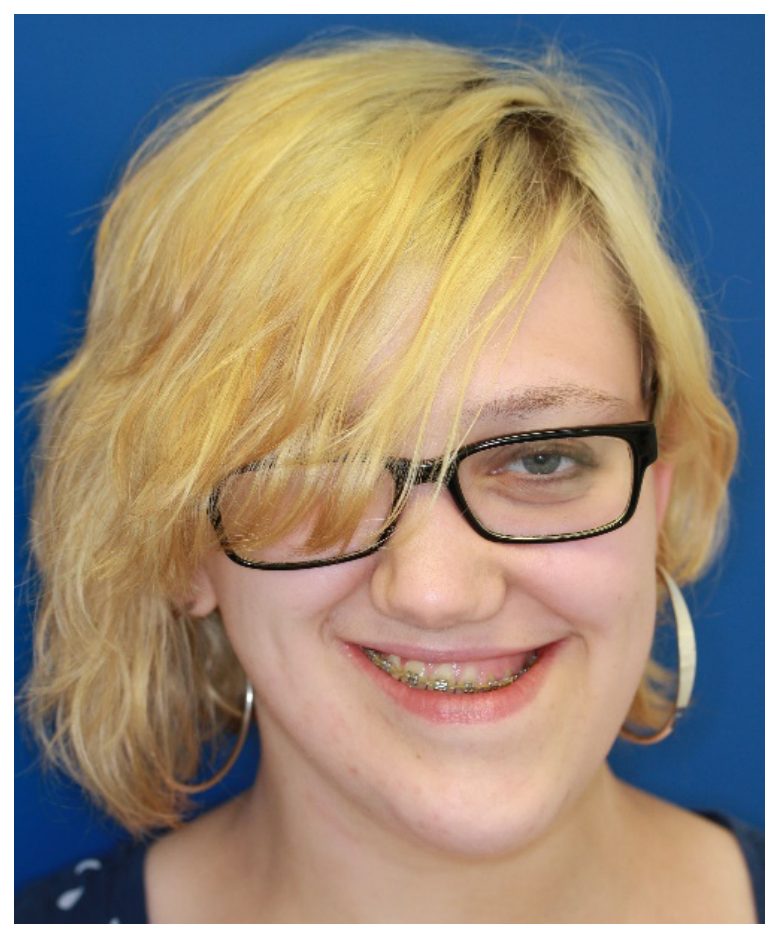

Figure 6c

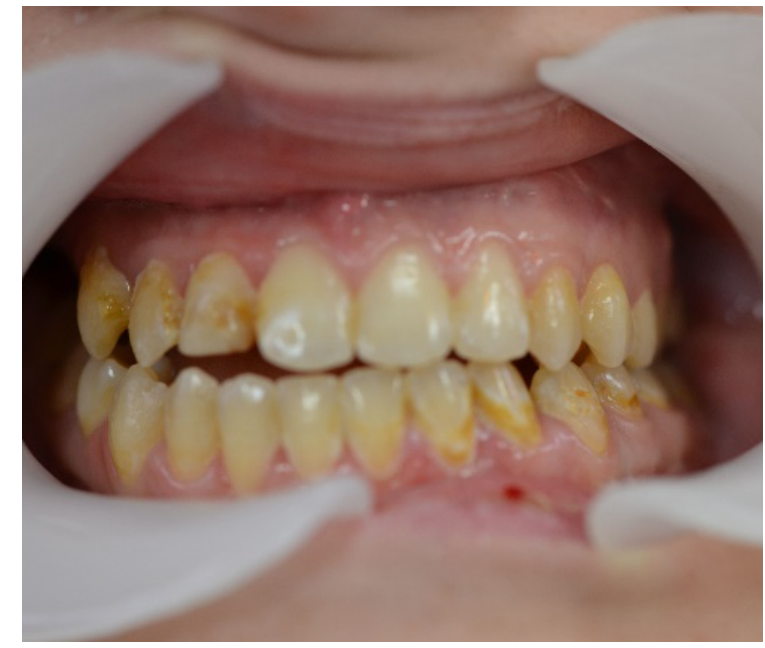

Figure $6 b$

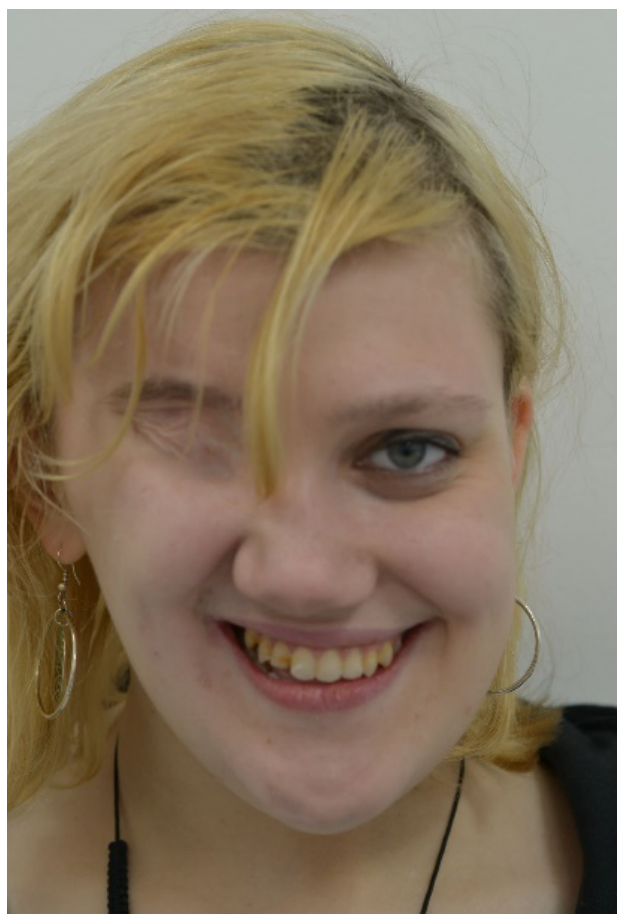

Figure 6d

Figure 6: a) Postoperative occlusion, LHS; b) Postoperative oral frontal view; c) Preoperative facial view; d) Postoperative facial view.

decreased blood flow to the corresponding regions is unlikely to be overcome by collateral blood supply which will form as development continues.

Following skeletal maturity, the patient presented with craniofacial microsomia-like characteristics including severe facial asymmetry, featuring orbital, Maxillary, and mandibular hypoplasia, and an asymmetric class III malocclusion with a severe occlusal cant. Orthognathic surgery was staged due to the significant asymmetric Maxillary advancement that would not have been possible in a one stage orthognathic procedure. The two step bimaxillary procedure allowed for gradual distraction osteogenesis of the Maxilla. Traditional distraction osteogenesis (DO) allows bone growth to occur in a gap between two vascularized bone segments [22]. Following initial osteotomy and a delay of approximately 5 days to allow a callous to form a tensile force is applied across the gap. This elongates the callous, bridging the two segments slowly and initiating osteogenesis and histogenesis in order to generate new vascularized bone spanning the gap [22]. The distraction process allows for slow growth of vasculature into the bony gap thereby negating the risk of inevitable a vascular necrosis that would occur in such a large movement of the Maxilla. In our procedure, a variation of this technique was utilized where the Maxilla was distracted on one side only in order to rotate the Maxilla and correct the significant dental centreline discrepancy. Supplementing the DO with an iliac 
crest bone graft reduces the possibility of surgical relapse and increases long term stability.

\section{Conclusion}

It is evident that the vascular insult from the ECA ligation at a young age, and long-term chemotherapy and radiation therapy played a significant role in the patient developing craniofacial microsomia and severe malocclusion. A two-stage orthognathic procedure was essential to correct the facial misalignment and functional irregularities. The two-stage process allowed for a more controlled corrective measure which minimizes the risk for a vascular necrosis while ensuring long term stability.

\section{References}

1. Stout AP, Murray MR (1942) Hemangiopericytoma: A vascular tumor featuring Zimmermann's pericytes. Annals of surgery 116: 26-33.

2. Enzinger FM, Smith BH (1976) Hemangiopericytoma: An analysis of 106 cases. Human Pathology 7: 61-82.

3. Ghose A, Guha G, Kundu R, et al. (2017) CNS Hemangiopericytoma: A systematic review of 523 patients. Am J Clin Oncol 40 223-227.

4. Fletcher CD (2014) The evolving classification of soft tissue tumours - an update based on the new 2013 WHO classification. Histopathology 64: 2-11.

5. Anand R, Gupta S (2010) Hemangiopericytoma of the maxilla in a pediatric patient: A case report. J Dent Child (Chic) 77: 180-182.

6. Hsu PY, Hsu WM, Huang HY, et al. (2006) Congenital hemangiopericytoma in a neonate. Journal of the Formosan Medical Association 105: 247-251.

7. Yamanishi T, Nishio J, Inoue M, et al. (2007) A case of congenital maxillary hemangiopericytoma: A case report. Journal of Oral and Maxillofacial Surgery 65: 549-552.

8. Ribeiro SF, Chahud F, Cruz AA (2012) Orbital hemangiopericyto$\mathrm{ma} / \mathrm{solitary}$ fibrous tumor in childhood. Ophthalmic Plast Reconstr Surg 28: e58-e60.

9. Wushou A, Miao XC, Shao ZM (2015) Treatment outcome and prognostic factors of head and neck hemangiopericytoma: Meta-analysis. Head \& Neck 37: 1685-1690.

10. Simmonds JC, Rebeiz EE (2017) Surgical resection of sinonasal hemangiopericytoma involving anterior skull base: Case reports and literature review. Am J Otolaryngol 38: 87-91.

11. Gomez-Rivera F, Fakhri S, Williams MD, et al. (2012) Surgical management of sinonasal hemangiopericytomas: A case series. Head \& neck 34: 1492-1496.

12. Serrano E, Coste A, Percodani J, et al. (2002) Endoscopic sinus surgery for sinonasal haemangiopericytomas. The Journal of Laryngology \& Otology 116: 951-954.

13. Nasr MM (2015) Bilateral external carotid artery ligation: A life saving procedure in severe maxillofacial trauma. Int J Surg Case Rep 8: 81-83.

14. Fanning NF, Kahn A, Corbally MT (1997) External carotid artery ligation for life-threatening hemorrhage in exsanguinating orbital facial congenital hemangiopericytoma. J Pediatr Surg 32: 1252-1254.

15. Bouloux GF, Perciaccante VJ (2009) Massive hemorrhage during oral and maxillofacial surgery: Ligation of the external carotid artery or embolization?. J Oral Maxillofac Surg 67: 1547-1551.

16. Gleysteen J, Troob S, Light T, et al. (2017) The impact of prophylactic external carotid artery ligation on postoperative bleeding after transoral robotic surgery (TORS) for oropharyngeal squamous cell carcinoma. Oral oncology 70: 1-6.

17. Pluijmers BI, Caron CJ, Dunaway DJ, et al. (2014) Mandiblular reconstruction in the growing patient with unilateral craniofacial microsomia: A systematic review. Int J Oral Maxillofac Surg 43: 286-295.

18. Bertin H, Mercier J, Cohen A, et al. (2017) Surgical correction of Mandiblular hypoplasia in hemifacial microsomia: A retrospective study in 39 patients. J Craniomaxillofac Surg 45: 1031-1038.

19. Moulin-Romsée C, Verdonck A, Schoenaers J, et al. (2004) Treatment of hemifacial microsomia in a growing child: The importance of co-operation between the orthodontist and the maxillofacial surgeon. J Orthod 31: 190-200.

20. Yamaguchi K, Lonic D, Ko EW, et al. (2017) An integrated surgical protocol for adult patients with hemifacial microsomia: Methods and outcome. PLoS One 12: e0177223.

21. Luo E, Yang S, Du W, et al. (2016) Bimaxillary orthognathic approach to correct skeletal facial asymmetry of hemifacial microsomia in adults. Aesthetic Plastic Surgery 40: 400-409.

22. Lucchese A, Albertini P, Asperio P, et al. (2018) Treatment of severe maxillary hypoplasia with combined orthodontics and distraction osteogenesis. J Craniofac Surg 29: 970-972.

DOI: $10.36959 / 605 / 540$

Copyright: (c) 2020 Rahman A, et al. This is an open-access article distributed under the terms of the Creative Commons Attribution License, which permits unrestricted use, distribution, and reproduction in any medium, provided the original author and source are credited. 